\title{
AN INVERSION FORMULA INVOLVING PARTITIONS
}

\author{
BY PETER DOUBILET \\ Communicated by Gian-Carlo Rota, July 25, 1972
}

In this note we outline a combinatorial proof of an inversion formula involving partitions of a number. This formula can be used to obtain the theory of symmetric group characters in a purely combinatorial way, as will be done in a forthcoming book, The combinatorics of the symmetric group, by the present author and Dr. G.-C. Rota.

The terminology we use is as follows. By a composition $\alpha$ of an integer $n$ we mean a sequence $\left(\alpha_{1}, \alpha_{2}, \ldots, \alpha_{s}\right)$ of nonnegative integers whose sum is $n$. A partition of $n$ is a composition $\lambda=\left(\lambda_{1}, \lambda_{2}, \ldots, \lambda_{p}\right)$ with $\lambda_{1} \geqq \lambda_{2}$ $\geqq \cdots \geqq \lambda_{p}>0$. The notation $\lambda \vdash n$ means " $\lambda$ is a partition of $n$ ". We use the symbols $\alpha, \beta$ for compositions, $\lambda, \mu, \rho$ for partitions.

A Young diagram of shape $\lambda$ is an array of dots, with $\lambda_{1}$ dots in the first row, $\lambda_{2}$ in the second row, etc., in which the first dots from the rows lie in a column, the second dots form a column, and so on. The conjugate partition $\tilde{\lambda}$ of $\lambda$ is the shape obtained when the Young diagram of shape $\lambda$ is transposed about its main diagonal, i.e., the rows of the transposed diagram are the columns of the original diagram. A generalized Young tableau (GYT) $\pi$ of shape $\lambda$ is an array of integers $q_{i j}(i=1,2, \ldots, p$, $\left.j=1,2, \ldots, \lambda_{i}\right)$ with $q_{i j}>0, q_{i, j+1} \geqq q_{i j}$ if $j<\lambda_{i}$, and $q_{i+1, j}>q_{i j}$ if $j \leqq \lambda_{i+1}$, i.e., an array of positive integers of shape $\lambda$ which is increasing nonstrictly along the rows and increasing strictly down the columns. The type of a GYT $\pi$ is the composition $\alpha=\left(\alpha_{1}, \alpha_{2}, \ldots, \alpha_{s}\right)$ of $n$ (where $\lambda \vdash n)$, where $\alpha_{i}$ is the number of times the integer $i$ appears in $\pi$.

If $\alpha=\left(\alpha_{1}, \ldots, \alpha_{s}\right)$ is a composition of $n$ with $s \leqq n$, and $\tau \in S_{n}$ (the symmetric group on $\{1,2, \ldots, n\})$, then $\tau \cdot \alpha$ is the composition of $n$ whose parts are $\alpha_{i}+\tau(i)-i, i=1,2, \ldots, n$ (where $\alpha_{i}=0$ if $i>s$ ), if all these parts are nonnegative, and $\tau \cdot \alpha$ is undefined otherwise. We also define $\tau * \lambda$ to be the partition of $n$ whose parts are $\lambda_{i}+\tau(i)-i$ in nonincreasing order if all these parts are nonnegative, and $\tau * \lambda$ is undefined otherwise.

Our inversion formula can now be stated.

THEOREM . Let $f, g$ be mappings from $\{\lambda \mid \lambda \vdash n\}$ to some field $F$ of characteristic 0 . Then

$$
f(\lambda)=\sum_{\tau \in S_{n}}(\operatorname{sign} \tau) g(\tau * \lambda) \leftrightarrow g(\lambda)=\sum_{\mu \vdash n} K_{\mu \lambda} f(\mu),
$$

AMS (MOS) subject classifications (1970). Primary 05A17; Secondary 05B20.

Key words and phrases. Composition, partition, conjugate partition, Young diagram, generalized Young tableau, partial ordering, incidence algebra. 
where $K_{\mu \alpha}$ is the number of GYT $\pi$ of shape $\mu$ and type $\alpha$.

It should be noted that this formula follows immediately from the fact that $e_{\lambda}=\sum_{\tau}(\operatorname{sign} \tau) h_{\tau * \lambda}$ and $h_{\lambda}=\sum_{\mu} K_{\mu \lambda} e_{\mu}$, where the $h$ 's and the $e$ 's are respectively the complete homogeneous symmetric functions and the Schur functions, since $\left\{h_{\lambda} \mid \lambda \vdash n\right\}$ and $\left\{e_{\lambda} \mid \lambda \vdash n\right\}$ are linearly independent sets. However, since one of our uses of the theorem is to prove just this connection between the $h$ 's and the $e$ 's, we want to prove the theorem from first principles. To do this we state a number of lemmas and outline some of their proofs.

Let $\leqq$ be the partial ordering on $\{\lambda \vdash n\}$ given by $\lambda \leqq \mu$ iff $\lambda_{1}+\lambda_{2}$ $+\cdots+\lambda_{i} \leqq \mu_{1}+\mu_{2}+\cdots+\mu_{i} \forall_{i}$.

LEMMA 1 . $K_{\lambda \mu} \neq 0$ implies $\lambda \geqq \mu$, and $K_{\lambda \lambda}=1$.

COROLlaRY. The matrix $\left(K_{\lambda \mu}\right)$ is nonsingular (with determinant 1$)$.

Let $m(\alpha, \beta)$ be the number of matrices with entries 0 and 1 with the sum of the $i$ th row being $\alpha_{i}$, the sum of the $j$ th column being $\beta_{j}$.

LEMMA 2. $m(\lambda, \mu)=\sum_{\rho} K_{\rho \lambda} K_{\tilde{\rho} \mu}$.

ProOF. Knuth's dual correspondence gives a constructive proof of this fact.

COROllaRY. The matrix $(m(\lambda, \mu))$ is nonsingular (with determinant \pm 1 ).

Proof. By Lemma $2,(m(\lambda, \mu))=\left(K_{\lambda \mu}\right)^{T} \cdot\left(K_{\tilde{\lambda} \mu}\right)$. $\left(K_{\lambda \mu}\right)$ has determinant 1 , and $\left(K_{\tilde{\lambda} \mu}\right)=P \cdot\left(K_{\lambda \mu}\right)$, where $P$ is the permutation matrix of the permutation $\lambda \rightarrow \tilde{\lambda}$ (i.e., $P=\left(p_{\lambda \mu}\right)$, with $p_{\lambda \mu}=1$ if $u=\tilde{\lambda},=0$ if $\mu \neq \tilde{\lambda}$ ).

LEMMA 3. $K_{\lambda_{\alpha}}=\sum_{\tau \in S_{n}}(\operatorname{sign} \tau) m(\tau \cdot \lambda, \alpha)$.

FIRST PROOF. We outline a proof as follows.

Let $M(\alpha, \beta)$ be the set of $0-1$ matrices with row sums $\left(\alpha_{1}, \alpha_{2}, \ldots\right)$, column sums $\left(\beta_{1}, \beta_{2}, \ldots\right)$. To $A \in M(\tau \cdot \lambda, \alpha)$, let $(t, i, j)$ be the least triple (ordered lexicographically) such that $i<j$ and $\rho_{i}^{(t)}-\tau(i)=\rho_{j}^{(t)}-\tau(j)$, where $\rho_{i}^{(t)}$ is the sum of the first $t$ entries in the $i$ th row of $A$, if any such triple exists. If $(t, i, j)$ exists, switch the first $t$ entries of the $i$ th row with those of the $j$ th and call the resulting matrix $B$. The following facts can be shown to hold for the correspondence $A \rightarrow B$.

(i) If $A \rightarrow B$ via the triple $(t, i, j)$ and $A \in M(\tau \cdot \lambda, \alpha)$, then $B \in M$ $((\tau \circ(i j)) \cdot \lambda, \alpha)$, so that the contributions of $A$ and $B$ in $\sum_{\tau \in S_{n}}(\operatorname{sign} \tau) m(\tau \cdot \lambda, \alpha)$ cancel each other out.

(ii) If $A \rightarrow B$ then $B \rightarrow A$.

(iii) No triple $(t, i, j)$ exists for $A \in M(\tau \cdot \lambda, \alpha)$ iff $\tau=1$ (= identity in $S_{n}$ ) and $\rho_{i}^{(t)}-i>\rho_{j}^{(t)}-j$ for all triples $(t, i, j)$ with $i<j$, or equivalently 
$\tau=1$ and $\rho_{i}^{(t)} \geqq \rho_{i}^{(t)} \forall(t, i, j)$ with $i<j$.

From (i), (ii), and (iii), it follows that $\sum_{\tau \in S_{n}}(\operatorname{sign} \tau) m(\tau \cdot \lambda, \alpha)$ is the number of $A \in M(\lambda, \alpha)$ satisfying the condition in (iii). Now to each such $A \in M(\lambda, \alpha)$ associate the array $\pi$ of shape $\tilde{\lambda}$ and type $\alpha$ letting the $k$ th column of $\pi$ consist of those $j$ such that $a_{k j}=1$ (where $A=\left(a_{i j}\right)$ ), ordered in increasing fashion down the column. It is not difficult to show that $\pi$ is a GYT, and that every GYT of shape $\tilde{\lambda}$ and type $\alpha$ arises in this way, proving the result.

SECOND PROOF. If $a_{k}$ is the elementary symmetric function of degree $k$ on variables $x_{1}, x_{2}, \ldots, x_{n}$ and if $\Delta=\operatorname{det}\left(x_{i}^{n-j}\right)$, we can obtain the equality in Lemma 3 by computing $a_{\alpha_{s}} \cdot a_{\alpha_{s-1}} \cdots a_{\alpha_{1}} \cdot \Delta$ in two different ways.

COROLlaRY. $K_{\tilde{\lambda} \alpha}=\sum_{\tau \in S_{n}}(\operatorname{sign} \tau) m(\tau * \lambda, \alpha)$.

Proof. $m(\alpha, \beta)$ does not depend on the order of the entries in the sequence $\alpha=\left(\alpha_{1}, \alpha_{2}, \ldots, \alpha_{s}\right)$, so $m(\tau * \lambda, \alpha)=m(\tau \cdot \lambda, \alpha)$.

It should be noted at this point that for the same reason as is given in the proof of the above corollary, it follows from Lemma 3 that $K_{\lambda \alpha}$ does not depend on the order of the entries in $\alpha$.

Finally, we prove the theorem.

PROOF OF THEOREM. It is easily shown that it suffices to find nonsingular matrices $\left(a_{\lambda \mu}\right),\left(b_{\lambda \mu}\right)$ such that $a_{\lambda \mu}=\sum_{\tau \in S_{n}}(\operatorname{sign} \tau) b_{\tau * \lambda, \mu}$ and $b_{\lambda \mu}=\sum_{\rho} K_{\rho \lambda} a_{\rho \mu}$. But by what we have proved already, we can take $a_{\lambda \mu}=K_{\lambda \mu}, b_{\lambda \mu}=m(\lambda, \mu)$, so we are done.

Note. This inversion formula is similar to a Möbius inversion, for it is equivalent to the fact that the functions $\phi(\lambda, \mu)=K_{\mu \lambda}$ and $\psi(\lambda, \mu)$ $=\sum_{\tau \in S_{n} \text { s.t. } \tau * \lambda=\mu}(\operatorname{sign} \tau)$ are inverse to each other in the incidence algebra of $\{\lambda \mid \lambda \vdash n\}$ with respect to the ordering $\leqq$.

\section{REFERENCES}

1. Philip Hall, The algebra of partitions, Proc. Fourth Canad. Math. Congr. (Banff 1957), 1959, pp. 147-159.

2. D. Knuth, Permutations, matrices, and generalized Young tableaux, Pacific J. Math. 34 (1970), 709-727. MR 42 \# 7535.

3. C. Schensted, Longest increasing and decreasing subsequences, Canad. J. Math. 13 (1961), 179-191. MR 22 \# 12047.

4. M. P. Schützenberger, Quelques remarques sur une construction de Schensted, Math. Scand. 12 (1963), 117-128. MR 32 \# 7433.

5. R. P. Stanley, Theory and applications of plane partitions. I, Studies in Appl. Math. 50 (1971), 167-188.

Department of Mathematics, Massachusetts Institute of Technology, Cambridge, MASSACHUSETTS 02139 